\title{
Elevation of Clavibacter michiganensis subsp. californiensis to species level as Clavibacter californiensis sp. nov., merging and re-classification of Clavibacter michiganensis subsp. chilensis and Clavibacter michiganensis subsp. phaseoli as Clavibacter chilensis sp. nov. based on complete genome in-silico analyses
}

\subsection{Author names}

Dario Arizala, Shefali Dobhal, Anne M. Alvarez and Mohammad Arif*

\subsection{Affiliation}

Department of Plant and Environmental Protection Sciences, University of Hawaii at Manoa, Honolulu, HI 96822

\subsection{Corresponding author}

arif@hawaii.edu; Phone: +1-808-956-7765

\subsection{Keyword}

Clavibacter; taxonomy; whole genome; ANI; dDDH; MLSA; phylogenomics

1.5 Repositories:

CP040786-СP040787; CP040788-СP040791; СP040792-СР040794; СР040795-СР040796; СР040797

\section{ABSTRACT}

The Gram-positive Clavibacter genus is currently divided into seven species (C. michiganensis, C. nebraskensis, C. capsici, C. sepedonicus, C. tessellarius, C. insidiosus and C. zhangzhiyongii) and three subspecies (C. michiganensis subsp. californiensis, C. 
michiganensis subsp. chilensis and C. michiganensis subsp. phaseoli). Recent studies have indicated that the taxonomic rank of the subspecies must be re-evaluated. In this research, we assessed the taxonomy position of the three C. michiganensis subspecies and clarified the taxonomic nomenclature of other 75 Clavibacter strains. The complete genomes of the type strains of the three Clavibacter subspecies, type strain of C. tessellarius and C. nebraskensis A6096 were sequenced using PacBio RSII technology. Application of whole-genome-based computational approaches such as average nucleotide identity (ANI), digital DNA-DNA hybridization (dDDH), multi-locus sequence analysis (MLSA) of seven housekeeping genes (acnA, atpD, bipA, icdA, $m t l D$, recA and $r p o B$ ), phylogenomic tree reconstructed from 1,028 core genes, and ANI-based phylogeny pinpointed conclusive evidence to raise $C$. michiganensis subsp. californiensis to the species status. These results led us to propose the establishment of $C$. californiensis sp. nov. as a species with its type strain $\mathrm{C}^{2} 5^{\mathrm{T}}(=\mathrm{CFBP}$ $8216^{\mathrm{T}}=\mathrm{ATCC}$ BAA-2691 ${ }^{\mathrm{T}}$ ). Moreover, the orthologous and in-silico dot plot analyses, along with the aforementioned bioinformatic strategies, revealed a high degree of homology between C. michiganensis subsp. chilensis and C. michiganensis subsp. phaseoli. Based on these outcomes, we proposed to combine both subspecies into a single taxon and elevate its rank to the species level as $C$. chilensis sp. nov., with ZUM3936 $\left(=\right.$ ATCC BAA-2690 $\left.{ }^{\mathrm{T}}=\mathrm{CFBP} 8217^{\mathrm{T}}\right)$ as the type strain.

\section{Introduction}

The genus Clavibacter, described by Davis et al. [1] constitutes a set of Gram-positive bacterial phytopathogens of agricultural crops, namely, tomato, pepper, potato, wheat, corn and alfalfa [2]. Originally, the genus Clavibacter was solely composed by the species C. michiganensis [3], which was divided into seven pathogenic subspecies assigned according to its narrow host specificity including: C. michiganensis subsp. michiganensis, the causal agent of canker and 
wilt of tomato [3, 4]; C. michiganensis subsp. sepedonicus, responsible of potato ring rot [2]; C. michiganensis subsp. insidiosus, inducing wilting and stunting in alfalfa [5]; C. michiganensis subsp. tessellarius, pathogenic to wheat, producing leaf freckles and leaf spots in a mosaic pattern [6]; C. michiganensis susbp. nebraskensis, causing blight and wilting of maize [7]; C. michiganensis susbp. capsici, inducing canker in pepper [8] and C. michiganensis subsp. phaseoli, reported as the responsible species of bacterial bean leaf yellowing [9]. The first three aforementioned subspecies have been categorized in the A2 list as quarantine organisms by the European and Mediterranean Plant Protection Organization [10]. Additionally, two subspecies isolated from tomato seeds, and described as non-pathogenic were named as C. michiganensis subsp. californiensis and C. michiganensis subsp. chilensis, based on their geographic origin California and Chile, respectively [11]. In 2018, five of the pathogenic subspecies were elevated to species level and reclassified as $C$. sepedonicus, $C$. insidiosus, C. tessellarius, C. nebraskensis and C. capsici based on whole-genome and multilocus sequence analyses [12]. Later, this new taxonomy assignation was also supported by a genome-based taxonomic study conducted in the phylum Actinobacteria and the description of C. michiganensis subsp. michiganensis, the bacterial tomato pathogen, was emended as $C$. michiganensis [13]. Most currently, a new pathogenic bacterium causing leaf brown spot and decline of barley was described as C. zhangzhiyongii [14].

Accurate systematics as well as a concise and reliable taxonomy designation of prokaryotes are highly primordial steps in epidemiology, pathogen surveillance, genome-informed diagnostics and for an efficient disease management $[15,16]$. Next generation sequencing has boosted the identification of unique genomic traits within a taxa and the development of novel wholegenome-based computational tools for bacterial taxonomy classification, namely: phylogenetic tree reconstruction using core-genome alignment [17] and species delineation through calculation of the Average Nucleotide Identity (ANI) [18, 19] and digital DNA-DNA 
hybridization $(\mathrm{dDDH})[20,21]$. Inclusion of these approaches in phylogenomics have elucidated novel species, and helped to clarify the taxonomy position of complex and highly heterogeneous bacterial species, which would have not been possible by using merely former taxonomic procedures, such as phenotypic characterization, 16S ribosomal RNA sequencing and multi-locus sequencing typing/analysis (MLST/MLSA) $[15,16]$. In Clavibacter, for instance, the use of high throughput taxonomic approaches allowed to reclassify some subspecies to species level [12], and to describe new species, namely C. michiganensis subsp. californiensis and subsp. chilensis [11]. However, when the above two subspecies were described, their respective genomes were not available in any database. In 2018, the draft genomes of these two isolates together with C. michiganensis subsp. phaseoli were published and available in the NCBI GenBank database [22]. Later, two comparative genomics studies, based on pairwise comparison analyses, showed ANI and dDDH values below the suggested 96- and $70-\%$ threshold for species delineation, respectively [23, 24], pinpointing that the subspecies status of these three organisms has to be re-evaluated. Considering this background, the main objectives of the present research were: first, to assess and validate the taxonomy status of the three subspecies, C. michiganensis subsp. californiensis, Clavibacter michiganensis subsp. chilensis and Clavibacter michiganensis subsp. phaseoli by using highquality complete genome sequences of their type strains, along with the complete genomes of the type strain of C. tessellarius and C. nebraskensis strain A6096; and second, to verify/correct the taxonomic description of other Clavibacter strains, whose assigned names in the NCBI database are incorrect, by using the modern whole-genome-based in silico tools for bacterial taxonomy description.

\section{Isolation, Ecology and Genome dataset preparation}


In this study, a large panel of 80 Clavibacter strains belonging to different species and isolated through different years, geographic regions, hosts, and niches were used for the phylogenomics and whole-genome-based computational analyses. Among the 80 strains, the complete genomes sequences of the type strains of the three subspecies of $C$. michiganensis (subsp. californiensis, chilensis and phaseoli), along with the type strain of $C$. tessellarius and $C$. nebraskensis strain A6096 were used for the taxonomy assessments instead of their previous draft genome versions. The bacterial cultures of these five strains were obtained from the Pacific Bacterial Collection at the University of Hawai'i at Manoa, Honolulu, HI, United States. The isolates were acquired from $-80^{\circ} \mathrm{C}$ and plated onto yeast sucrose calcium carbonate (YSC) medium (yeast extract $10 \mathrm{~g} / \mathrm{l}$, sucrose $20 \mathrm{~g} / \mathrm{l}$, calcium carbonate $20 \mathrm{~g} / \mathrm{l}$ and agar $17 \mathrm{~g} / \mathrm{l}$ ) and incubated for $72 \mathrm{~h}$ at $26^{\circ} \mathrm{C}\left( \pm 2^{\circ} \mathrm{C}\right)$. Complete or draft genomes of the other 75 Clavibacter strains were retrieved from the NCBI GenBank database as of October $13^{\text {th }}, 2021$. Detail descriptions of all strains used in this research are provided in Table S1.

\section{Phylogeny based on Multi-Locus Sequence Analysis (MLSA)}

To determine the taxonomic position of the five Clavibacter genomes sequenced in this study and resolve the taxonomy status of the other 75 Clavibacter strains listed in Table S1, a multilocus sequence analysis (MLSA) was performed using seven housekeeping genes acnA, atpD, bipA, icdA, $m t l D$, recA and rpoB. Multiple gene sequences were aligned using MUSCLE and then concatenated in Geneious Prime version 2021.1.1. The concatenated alignment was used to generate a maximum-likelihood (ML) phylogenetic tree based on a bootstrap test of 1,000 replicates. Phylogenetic evolutionary relationships were assessed in MEGA 11. All sequences of the seven housekeeping genes corresponding to the 75 Clavibacter strains were retrieved from complete or draft genomes available at the NCBI GenBank database (Table S1). Rathayibacter iranicus NCCPB2253 and Rathayibacter toxicus WAC3373 were used as 
outgroups for the phylogenetic analyses. On the phylogenetic tree (Fig 1), nine clear clusters were identified. The biggest and first cluster comprised 44 strains of the recently emended species C. michiganensis [13] and the second cluster grouped the type strain of the proposed species C. californiensis sp. nov. together with the strain AY1B2, isolated from ryegrass in 2013 and reported as C. michiganensis in the NCBI GenBank database. Following these two clusters, the third cluster harbored 2 sub-clades, the first composed of the strains VKM Ac2886, isolated from Sambucus racemosa, and CFBP 7491, isolated from tomato seeds; the second sub-clade integrated the type strains of C. michiganensis subsp. chilensis CFBP $8217^{\mathrm{T}}$, isolated from tomato seeds, and C. michiganensis subsp. phaseoli LPPA 982 ${ }^{\mathrm{T}}$, pathogen of bean, highlighting that these two subspecies must be merged into a single species. We proposed the species name for these 4 strains within the third cluster as C. chilensis sp. nov. Clusters fourth to sixth constituted monophyletic clades comprising the species $C$. nebraskensis, $C$. insidious and C. sepedonicus. Our C. nebraskensis strain A6096 positioned close to the strains HF4 and CFBP 7577. Interestingly, the strain CFBP 7494, a tomato endophytic bacterium isolated from seeds in Chile, clustered closely to C. insidiosus, like previous studies [25], but in a different sub-clade. Clusters seventh and eight encompassed the pathogens of pepper $(C$. capsici) and barley (C. zhangzhiyongii), respectively. The endophytic strain CFBP 7576 submitted in the NCBI database as C. michiganensis, grouped with other C. capsici isolates, which is coherent with the fact that this bacterium was proved to cause disease on pepper [25]. The last cluster comprised the strains DOAB 609, CFBP 8017, the type strain of C. tessellarius and CFBP 3399. Intriguingly, CFBP 3399, named as C. michiganensis and isolated from tulip (Tulipa sp.) in Netherlands, formed its own clade with C. tessellarius type strain ATCC $33566^{\mathrm{T}}$. We hypothesized that CFBP 3399 might be a pathogen of wheat similarly like the tomato endophyte CFBP 8017 that was observed to cause mosaic disease symptoms on wheat [25]. Although DOAB 609 and CFBP 8017 are closely related to ATCC 33566 ${ }^{\mathrm{T}}$, former analysis 
based on genome-to-genome similarity indices has shown that these strains should not be classified as C. tessellarius but as new species [23]. Indeed, our analyses, described further, match with these previous assumptions.

\section{Genome Features}

To provide a correct taxonomic delineation of the remaining Clavibacter subspecies, the complete genomes of type strains of C. michiganensis subsp. californiensis, C. michiganensis subsp. chilensis, C. michiganensis subsp. phaseoli, along with the type strain of C. tessellarius and C. nebraskensis strain A6096 were sequenced using PacBio RS II (Pacific Biosciences Inc., Menlo Park, CA, United States) powered by single-molecule real-time (SMRT) technology. Genome sequencing was carried out at the Washington State University facility. For whole genomic DNA extraction, a single colony corresponding to each Clavibacter strain was streaked on YSC medium [11] and grown overnight at $28^{\circ} \mathrm{C}$. Later, half loopful of pure bacterial culture was picked and genomic DNA was extracted using the QIAGEN Genomictip 100/G (Qiagen, Valencia, CA, United States) following the manufacturer's instructions. DNA was re-suspended into 300-350 $\mu \mathrm{L}$ TE (Tris-EDTA) buffer $\mathrm{pH}$ 8.0. Quantification and quality of DNA was analyzed by using NanoDrop spectrophotometer, Qubit 4, and by electrophoresis on $1.5 \%$ agarose gels. Genomic DNA libraries were prepared with a $20 \mathrm{~kb}$ insert size and sequenced using P6 polymerase enzyme and C4 sequencing chemistry. Prior assembly, long sequencing reads were trimmed according to the length and quality to obtain highly accurate long reads. Final assembly was done using the Hierarchical Genome Assembly Process (HGAP) version 4.0 (Pacific Biosciences, SMRT Analysis Software v2.3.0). The generated high-quality complete genomes of the five Clavibacter strains were annotated in three different pipelines: the NCBI Prokaryotic Genome Annotation Pipeline (PGAP) version 4.8 [26] the Integrated Microbial Genomes annotation pipeline version v.5.0.3 [27, 28] from 
the Joint Genome Institute (IMG-JGI) and the Rapid Annotation System Technology (RAST) server [29]. All genomes were deposited in the NCBI GenBank genome database with the accession numbers: CP040792-CP040794 (C. michiganensis subsp. californiensis CFBP 8216 ${ }^{\mathrm{T}}$ CP040795-CP040796 (C. michiganensis subsp. chilensis CFBP 8217T), CP040786CP040787 (C. michiganensis subsp. phaseoli LPPA 982'), CP040788-CP040791 (C. tessellarius ATCC 33566 ${ }^{\mathrm{T}}$ ) and CP040797 (C. nebraskensis A6096).

The genomes size of C. michiganensis subsp. californiensis CFBP $8216^{\mathrm{T}}$, C. michiganensis subsp. chilensis CFBP $8217^{\mathrm{T}}$, C. michiganensis subsp. phaseoli LPPA 982 ${ }^{\mathrm{T}}$, C. tessellarius ATCC $33566^{\mathrm{T}}$ and C. nebraskensis A6096 consisted of 3,255,537 bp with 3,032 coding sequences (CDS), 3,221,227 bp with 3,016 CDS, 3,22,6754 with 3,021 CDS, 3,366,694 with 3,145 CDS and 3,069,018 with 2,884 CDS, respectively. All five genomes contained six rRNA genes ( 2 copies of $5 \mathrm{~S}$ rRNA, 2 copies of $16 \mathrm{~S}$ rRNA and 2 copies of 23S rRNA), 45 tRNA genes and three non-coding RNA genes. The DNA G+C contents of the genomes were $72.78 \%$, $73.43 \%, 73.42 \%, 73.63 \%$, and $72.97 \%$, respectively. Strains CFBP $8216^{\mathrm{T}}$ harbored 2 plasmids of 78,006- and 20,294-bp while strains CFBP $8217^{\mathrm{T}}$ and LPPA $982^{\mathrm{T}}$ presented a single plasmid of 117,510- and 123061-bp, respectively. ATCC $33566^{\mathrm{T}}$ harbored three plasmids with lengths of 45,791-, 37,356- and 29,539-bp. No plasmid was found in C. nebraskensis A6096. Detail description of different genome features for each of the five bacterial strains sequenced in this study are provided in Table 1.

The average nucleotide identity (ANI) measures the genetic relatedness between a given set of genomes, and it is an important whole genome-based in silico tool applied extensively to define species boundaries among prokaryotic genomes $[18,19,30]$. Conversely, the digital DNADNA hybridization (dDDH) represents the genome-to-genome distances between complete or incomplete genomic sequences, and it has emerged as a potential bioinformatic method that emulates the traditional wet-lab DNA-DNA hybridization (DDH) employed in prokaryotes 
taxonomy [21, 31]. In this study, pairwise genome comparisons using ANI tool were computed using the CLC Genomics Workbench 21.0.5 (Qiagen, Germantown, MD) whereas the dDDH was measured using the genome-to-genome distance calculator (GGDC) 3.0 server (http://ggdc.dsmz.de/ggdc.php\#, [32]) with the recommended settings: BLAST+ as local alignment tool and formula 2, applied when incomplete genomes are submitted to the server and which consists on the sum of identities within high-scoring segment pairs (HSPs) per total HSP length [21]. ANI and dDDH values were combined in a single matrix and visualized as a heatmap (Figure 1) using the web-tool DISPLAYR (https://www.displayr.com/). The analysis included the five genomes sequenced in this study, along with 15 genomes of the other Clavibacter species with their respective type strains. To assign a correct taxonomy status for the remaining Clavibacter subspecies along with the other strains examined in this article, the species-delineation framework was set to $96 \%$ for ANI $[18,19]$ and $70 \%$ for dDDH [33]. Tables S2 and S3 show the ANI and dDDH values calculated among 79 Clavibacter strains. The strain CFBP $2404^{\mathrm{T}}$ was not included in the analyses since this isolate is the same type strain LMG $3663^{\mathrm{T}}$.

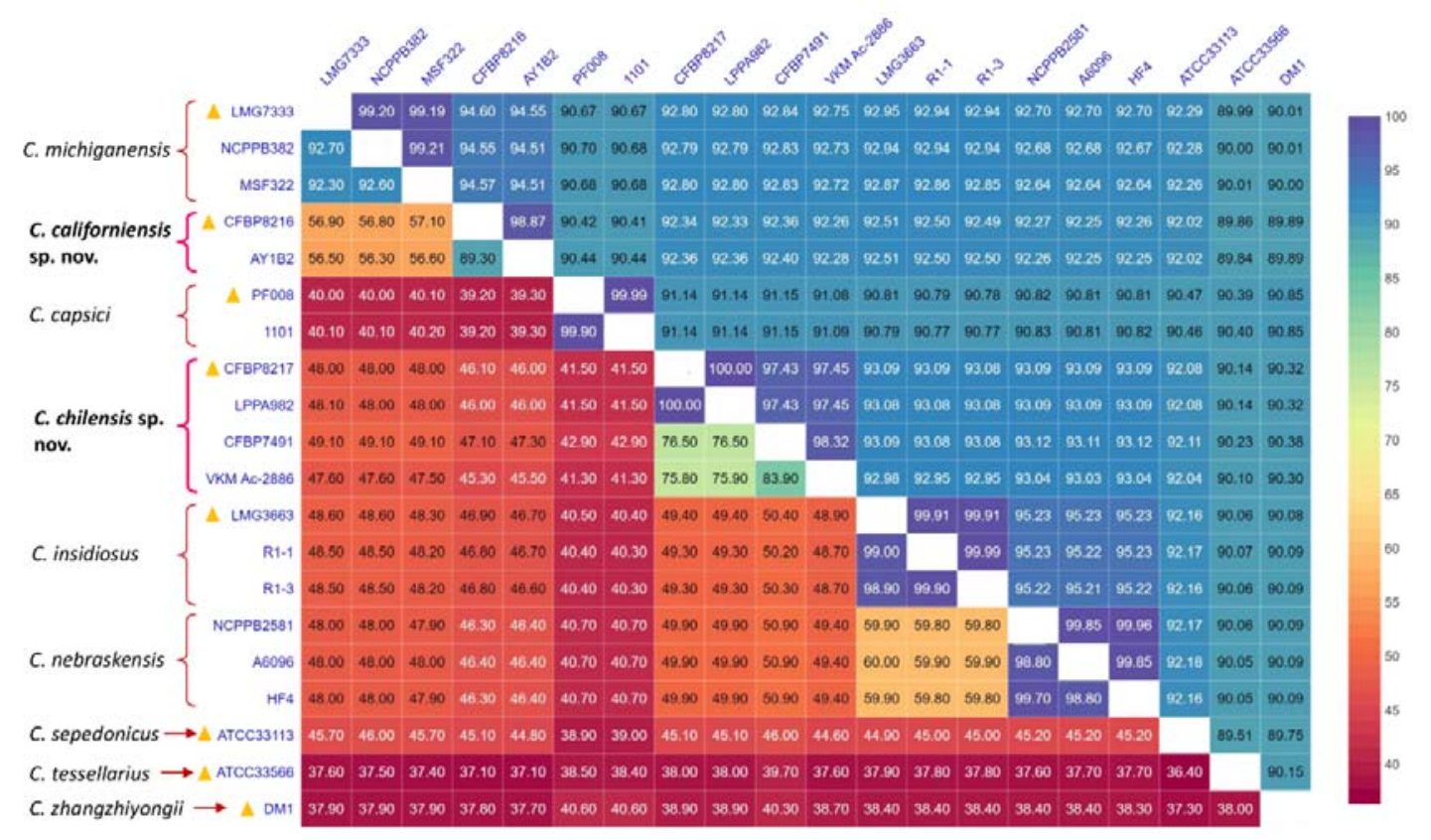


Figure 1. Combined average nucleotide identity (ANI, upper diagonal) and digital DNA-DNA hybridization (dDDH, lower diagonal) heatmap among all Clavibacter species. Cut-off values for species delineation are $96 \%(\mathrm{ANI})$ and $70 \%(\mathrm{dDDH})$.

Overall, pairwise ANI and dDDH values among all Clavibacter species were lower than the recommended species threshold of $96 \%[18,19]$ and $70 \%$ [33], respectively, supporting the elevation of some subspecies to the species level [12]. When comparing with other Clavibacter species, C. tessellarius and C. zhangzhiyongii were the most divergent species within the Clavibacter genus with the lowest ANI value of $90 \%$ for both, and dDDH of $38 \%$ and $40 \%$, respectively. Interspecies ANI numbers were observed less than $91 \%$ and $90 \%$ for pepper and potato pathogens, C. capsici and C. sepedonicus, respectively, whereas the minimum dDDH calculations were $38 \%$ and $36 \%$, respectively. In agreement with the MLSA phylogenetic tree, C. nebraskensis and C. insidiosus showed to be closely related since a high ANI of $95 \%$ and a dDDH of $60 \%$ were obtained between these two species. Moreover, the minimal interspecies ANI/dDDH values for C. sepedonicus, C. insidiosus and C. nebraskensis were 92-/46-, 91/40- and 91-/41-\%, respectively. The highest ANI and dDDH values between C. michiganensis and the other Clavibacter species or subspecies were $94.5 \%$ and $56.3 \%$, respectively, below the proposed $96 \%$ ANI and $70 \% \mathrm{dDDH}$ for the bacterial species boundary, corroborating the recent emended nomenclature of this species [13]. Clavibacter michiganensis subps. californiensis CFBP $8216^{\mathrm{T}}$ exhibited ANI and $\mathrm{dDDH}$ values of $98.87 \%$ and $89.3 \%$, respectively, with respect to the strain AY1B2, isolated from ryegrass, indicating that these two strains belong to the same species. On the other hand, the ANI values of CFBP $8216^{\mathrm{T}}$ and AY1B2 with the other species within the Clavibacter genus varied from $94.60 \%$ to $89.84 \%$, while dDDH values ranged 57.10- to $37.10-\%$, which were below the cut-off parameters for species delineation, 96\% ANI and 70\% dDDH, revealed that C. michiganensis subsp. 
californiensis must be elevated at the species level, and also, the taxonomic name of strain AYB2, wrongly described as C. michiganensis in the NCIBI database, must be corrected to C. californiensis sp. nov. Intriguingly, the type strains of C. michiganensis subsp. chilensis CFBP $8217^{\mathrm{T}}$ and C. michiganensis subsp. phaseoli LPPA $982^{\mathrm{T}}$ shared the highest nucleotide similarity of $100 \%$ for both ANI and $\mathrm{dDDH}$, indicating that these two bacteria must be merged as a single species, as suggested in previous publications $[23,24]$. In addition, ANI and dDDH analyses of strains CFBP 7491, VKM Ac-2886 with CFBP $8217^{\mathrm{T}}$ and LPPA $982^{\mathrm{T}}$ revealed average values above the cut-off for species delineation (ANI 98\%; dDDH 78\%), indicating that all four strains belong to the same species. Interspecies ANI and dDDH values between these four isolates and all other Clavibacter species ranged $93-90 \%$ and $50-38 \%$, respectively, which are below the edge of species threshold, denoting that these strains represent a different species within the genus Clavibacter. Hence, we proposed the name of $C$. chilensis sp. nov. with CFBP $8217^{\mathrm{T}}$ as the type strain, as formerly published by Yasuhara-Bell and Alvarez [11]. Although C. michiganensis subsp. phaseoli LPPA $982^{\mathrm{T}}$ was reported as the causal agent of bean leaf yellowing [9], no symptoms were induced in recent pathogenicity assays performed by Osdagui et al. [23] using this pathogen and C. michiganensis subsp. chilensis CFBP $8217^{\mathrm{T}}$ on common bean (cv. Red Kideny, cv. Pinto and cv. Navy), cowpea, mung bean, tomato, and pepper. Since both CFBP $8217^{\mathrm{T}}$ and LPPA $982^{\mathrm{T}}$ were demonstrated to be the same species [23, 24, this study] and CFBP $8217^{\mathrm{T}}$ was named based on its geographic origin of isolation instead of the host like LPPA $982^{\mathrm{T}}$, we consider that $C$. chilensis sp. nov. is the best appropriate name for describing the four above discussed strains, which have been isolated from distinct hosts such as tomato seeds (CFBP $8217^{\mathrm{T}}$ and CFBP 7491), bean seeds (LPPA 982 $)$ and red elderberry (VKM Ac-2886).

Non-pathogenic and seed-associated Clavibacter, tomato strains, have shown a wide genetic diversity, and phylogenetically different from the pathogenic C. michiganensis strains $[11,25$, 
34-36]. Moreover, several strains isolated from surface-disinfected tomato parts have been frequently described as endophytic Clavibacter strains with no disease symptoms, but shown colonization inside the tomato vascular tissues [2, 25]. Thapa et al. [25] reported five endophytic tomato Clavibacter strains (CASJ009, CFBP 7494, CFBP 7576, CFBP 8017, and CFBP 8019) — based on phylogenomic analysis, the strains displayed high heterogeneity and distributed in different clades; and particularly, CFBP 7494, CFBP 7576 and CFBP 8017 clustered with the alfalfa, pepper and wheat Clavibacter strains, respectively. Stem canker and mosaic disease symptoms were observed on pepper and wheat plants when inoculated with strains CFBP 7576 and CFBP 8017, respectively [25]. Here, we have assessed the taxonomy position of these three isolates (Tables S2 and S3). Intraspecies ANI (98\%) and dDDH (80\%) analysis demonstrated that CFBP 7576 belongs to C. capsici. Conversely, strains CFBP 7494 and CFBP 8017 exhibited ANI values of $96 \%$ (on the edge of species delineation) and $94.9 \%$, respectively, whereas the $\mathrm{dDDH}$ values $(64.6 \%$ and $57.7 \%$, respectively) were lower than the limit for species delineation after comparing with the type strains of $C$. insidiosus and $\mathrm{C}$. tessellarius, respectively,- - demostrated that both bacteria are closely related to the alfalfa and wheat strains, but genetically constitute novel species. This is in sync with our MLSA results, where both isolates formed a separate like-outgroup clade within $C$. insidiosus and $C$. tessellarius clusters (Figure 2). Interestingly, the ANI value between strain DOAB 609, described as C. tessellarius in the NCBI database, and C. tessellarius ATCC $33566^{\mathrm{T}}$ was $93.17 \%$, while $\mathrm{dDDH}$ value was $49 \%$; indicating that the taxonomic name of this strain is incorrectly assigned. Moreover, DOAB 609 showed to be closely related to the tomato endophyte CFBP 8017 based on our phylogenetic analysis (Figure 2); however, the ANI and dDDH data ( $92.78 \%$ and $47.2 \%$, respectively) between these strains revealed they are different species. Additionally, ANI and dDDH values between DOAB 609 and other Clavibacter members ranged $89-90 \%$ and $36.2-39.5 \%$, respectively, highlighted that this strain is a novel 
Clavibacter species. On the other hand, ANI and dDDH scores well above the species threshold (98.57\% and 85.4\%, respectively) were calculated between CFBP 3399, isolated from wild tulip, and the type strain of $C$. tessellarius, corroborating that the tulip strain belongs to the species C. tessellarius, which is coherent with the obtained MLSA phylogeny (Figure 2).

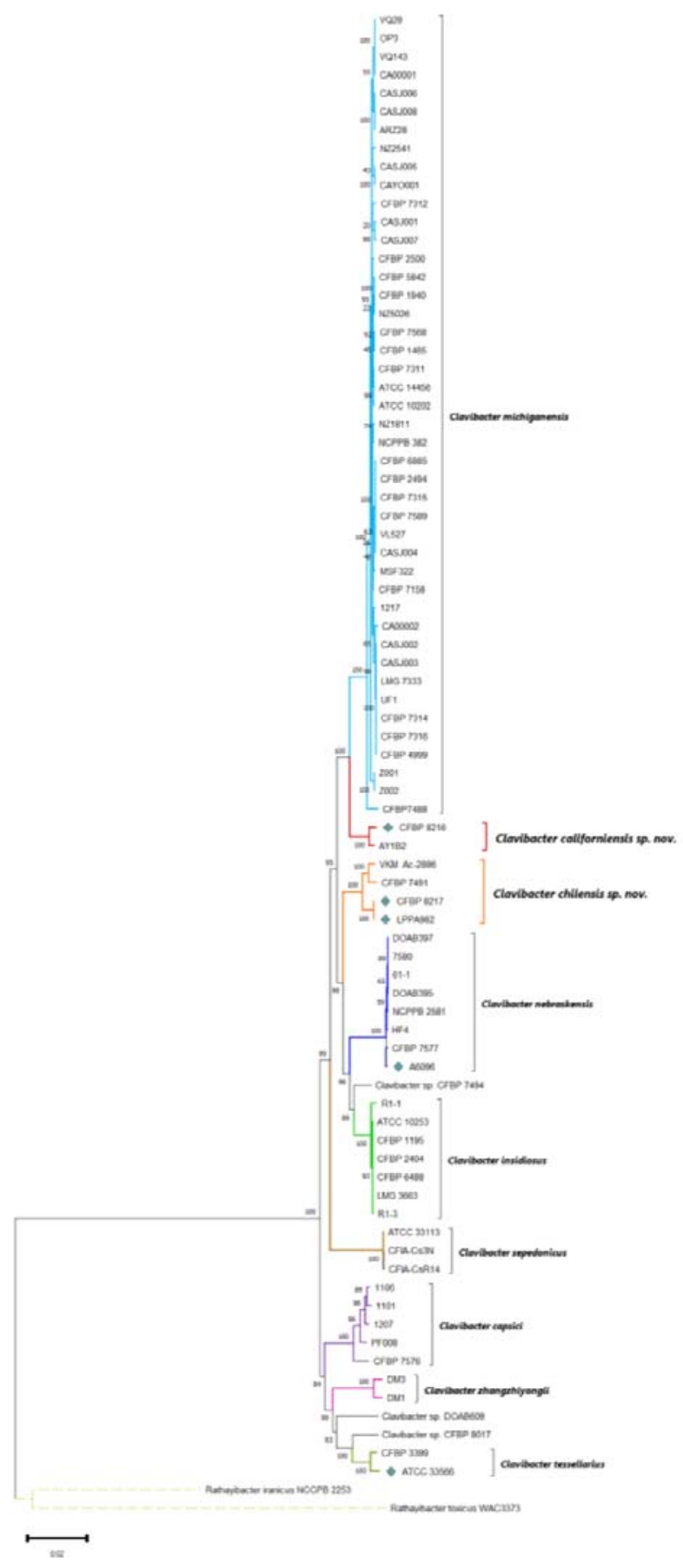


Figure 2. Multi-Locus Sequence Analysis (MLSA) based on the concatenated alignment of seven housekeeping genes, acnA, atpD, bipA, icdA, mtlD, recA, and rpoB. The maximumlikelihood (ML) phylogenetic tree was created using 80 Clavibacter strains with a bootstrap test of 1,000 replicates. Bootstrap values are indicated at branch points. Evolutionary distances were estimated by applying the Maximum Composite Likelihood (MCL) approach. There was a total of 13,154 positions (alignment length) in the final dataset. The tree was drawn to scale, with branch lengths measured in the number of substitutions per site. The branches were colorcoded to emphasize the clusters of each species. The genomes of the five Clavibacter strains sequenced in this study are pointed out with a teal diamond. Rathayibacter iranicus NCPPB 2253 and R. toxicus WAC3373 served as outgroups to root the tree. The evolutionary analysis was conducted in MEGA 11.

To further examine the taxonomy position of a large panel of Clavibacter strains including the five genomes sequenced here, a phylogenetic tree based on 1,028 core genes was generated (Figure 3). The FASTA genome files of 78 Clavibacter strains were re-annotated using the Rapid prokaryotic genome annotation pipeline Prokka [37]. The genome of C. nebraskensis CFBP 7577 was excluded due to the excessive number of contigs and to avoid interferences during the identification of core genes. The annotated GFF3 assemblies obtained using Prokka were applied to perform a pan-genome analysis using the ROARY pipeline [38]. A multiFASTA alignment based on all core genes derived from the pan-genome was created using PRANK [39]. The core-genome alignment served to construct a maximum likelihood (ML) phylogenetic tree using RAxML-NG [40] with a General Time-Reversible GAMMA model and bootstrap test of 1,000 replicates. Lastly, the phylogenetic ML tree was plotted, midpoint rooted, arranged according to increase order of nodes and color-coded using FigTree v1.4.4 (http://tree.bio.ed.ac.uk/software/figtree/). 


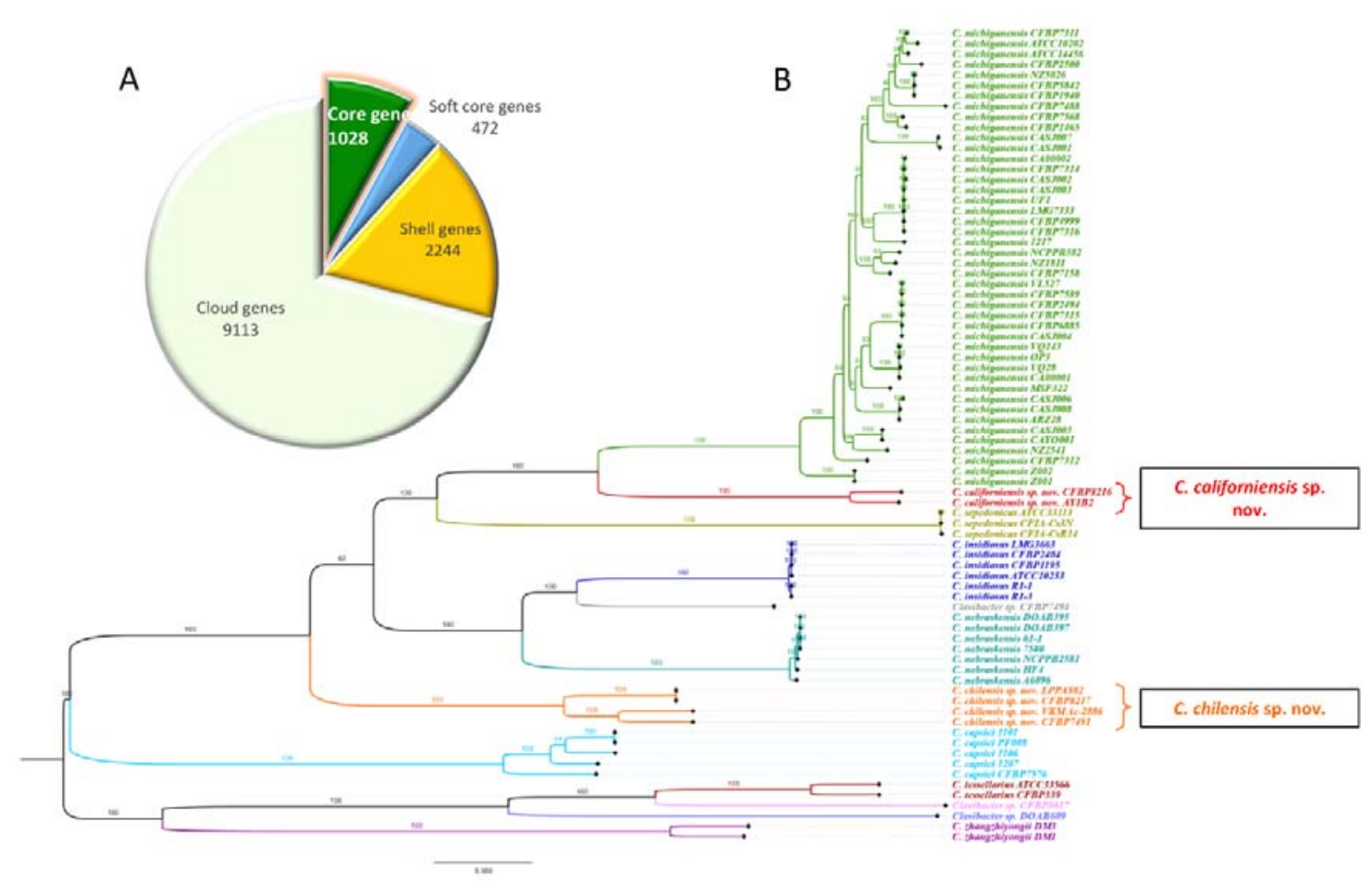

Figure 3. Maximum-likelihood (ML) phylogeny generated from the core-genome of 78 Clavibacter strains. (A) Pie chart depicting the proportion of core and accessory genes among the 78 Clavibacter genomes as predicted by the ROARY pipeline. (B) ML phylogentic tree reconstructed from the concatenated alignment of 1,028 core genes. A bootstrap test of 1,000 replicates was used to assess the statistical support of each node. The ML analysis was performed using the RAxML-NG tool with a General Time-Reversible GAMMA model. The phylogenetic tree was mid-point rooted and illustrated based on the increasing order of nodes. All strains and their respective branches of each Clavibacter species are highlighted with specific colors. The proposed species are pinpointed in a black rectangular frame. FigTree v1.4.4 was used to visualize and color-coded the ML tree.

In sync with the MLSA analysis, the core-genome phylogeny showed 9 clusters that represented each of the Clavibacter species. The cluster of $C$. californiensis sp. nov. included the type strain CFBP $8216^{\mathrm{T}}$ and the ryegrass strain AY1B2. Even though C. californiensis sp. 
nov. positioned closely to the C. michiganesis group, C. californiensis formed its own distinct and exclusive cluster, thus supporting their elevation to species level. The type strains of $C$. michiganensis subsp. chilensis CFBP $8217^{\mathrm{T}}$ and C. michiganensis subsp. phaseoli LPPA $982^{\mathrm{T}}$ clustered far away from the tomato pathogen strains - the branches of both strains converged from the same node, corroborating our previous analysis that this both subspecies must be elevated to the species level and merged as single species, for which we proposed the name $C$. chilensis sp. nov [11]. Additionally, the seed-associated tomato strain CFBP 7491 and the strain VKM Ac-2886 grouped together with CFBP $8217^{\mathrm{T}}$ and LPPA $982^{\mathrm{T}}$, revealing that $C$. chilensis sp. nov. harbors both pathogenic and endophytic strains isolated from different hosts. Importantly, the reconstructed core-genome ML tree confirmed that the tulip strain CFBP 3399 belongs to C. tessellarius since the nodes of this strain, along with ATCC $33566^{\mathrm{T}}$ clustered together. Moreover, an outgroup-like pattern was observed for the nodes of strains CFBP 7494 and CFBP 8017, which positioned close to C. insidiosus and C. tessellarius, showing that these strains are different species but are related to the alfalfa and wheat strains, respectively. In concordant with the MLSA, ANI and dDDH data, strain DOAB 609 clustered in its own clade and a bit distant from C. tessellarius ATCC $33566^{\mathrm{T}}$ and CFBP 8017, corroborating that DOAB 609 is misnamed as C. tessellarius; and hence, a correct name must be given to this new species. An identical clustering pattern of well-defined clades containing each of the Clavibacter species was visualized in the ANI-based phylogeny (Figure 4) derived from the computed ANI (Table S2), emphasizing the proposal of C. californiensis sp. nov. and C. chilensis sp. nov. as species. The ANI phylogenetic tree was built from the whole-genome alignment of 79 Clavibacter strains using the neighbor joining method with a bootstrap test of 1,000 replicates (Figure 4). The genome of $R$. toxicus WAC3373 was used as an outgroup and the phylogenetic analysis was generated in the CLC Genomics Workbench v.21.0.5. 


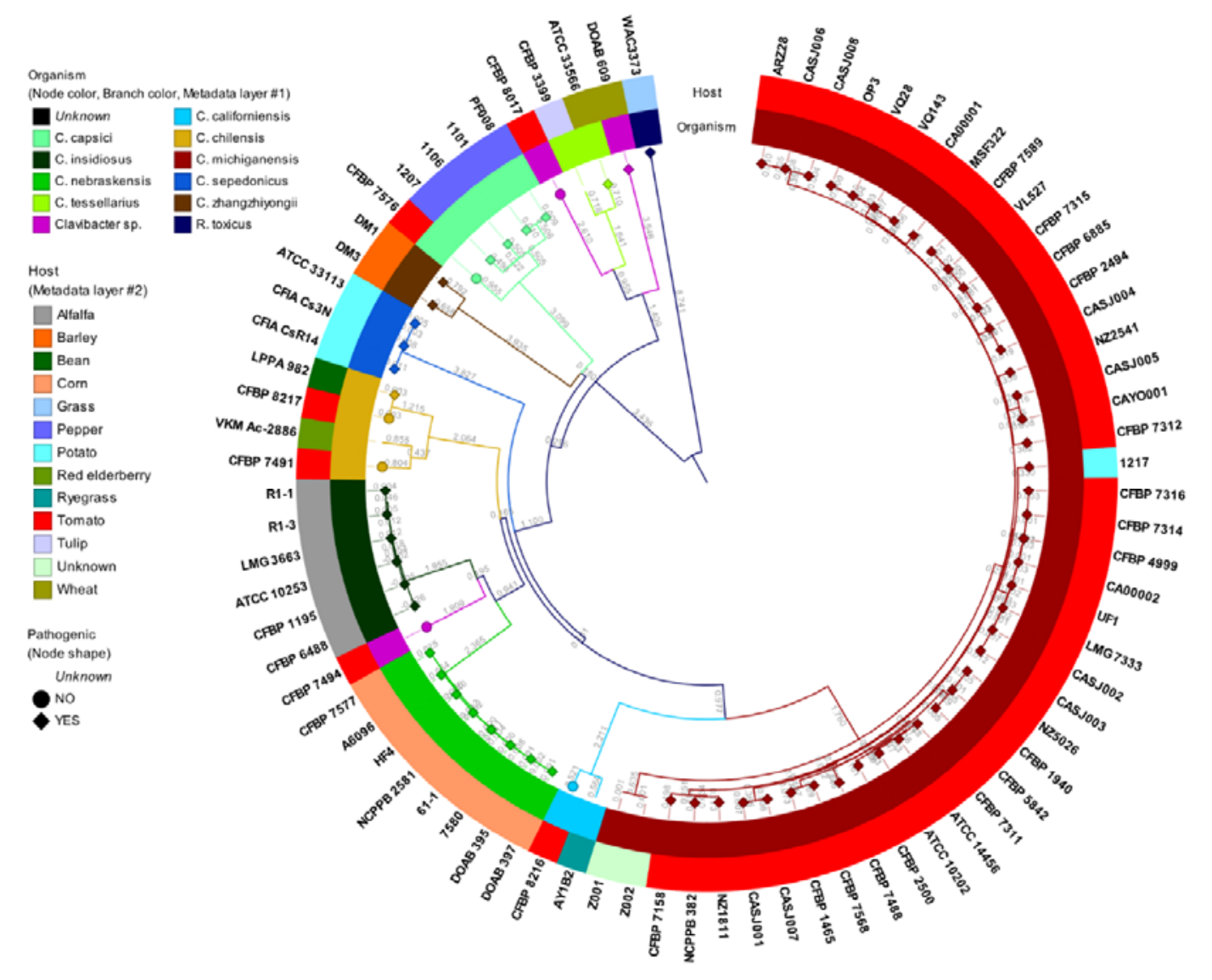

Figure 4. Whole-genome phylogeny based on the computed average nucleotide identity (ANI) of 79 Clavibacter strains. The neighbor joining tree was created using the CLC Genomics Workbench v.21.0.5 with a bootstrap test of 100 replicates. Rathayibacter toxicus WAC3373 was used as an outgroup to root the tree. The legends of layers corresponding to the organism and host, along with the node shape, are indicated on the left side of the graphic.

The type strain genome server (TYGS; https:/tygs.dsmz.de/) was used to confirm the taxonomy status of strains CFBP 7576, CFBP 7491, VKM Ac-2886, CFBP 7494, CFBP 8017 and DOAB 609. TYGS is a high-throughput platform that implements the techniques of the GGDC and compares the submitted genomes with the most curated, updated and valid database of type strain genomes $[32,41]$. The web server not only performs a phylogenomic analysis based on the computed $\mathrm{dDDH}$, but also it allows to delineate species according to the 
established $70 \% \mathrm{dDDH}[32,41]$; and hence, this is a reliable approach for microbial classification. In correlation with our previous analysis, TYGS categorized the strains CFBP 7494, CFBP 8017 and DOAB 609 as potential new species while strain CFBP 7576 was presented as C. capcisi and strains CFBP 7491 and VKM Ac-2886 were identified as C. michiganensis subsp. chilensis (here proposed as C. chilensis sp. nov.). A more elaborated taxonomy study is required to provide the most idoneous distinctive name for the strains CFBP 7494, CFBP 8017 and DOA B609, inferred here as novel Clavibacter species.

\section{Orthologous Analysis}

Since C. michiganensis subsp. chilensis CFBP $8217^{\mathrm{T}}$ and C. michiganensis subsp. phaseoli LPPA $982^{\mathrm{T}}$ shared the highest nucleotide identity in the ANI and $\mathrm{dDDH}$ (both $100 \%$ ) pairwise analyses, we performed a dedicated comparative orthologous assessment using the OrthoMCL pipeline v1.4 [42] between these two strains. Homologous protein sequences were computed based on an all-against-all BLASTp search approach with an E-value threshold $<1 \times 10^{-05}, 90 \%$ similarity and an alignment coverage $>70 \%$. The precalculated sequence similarity matrix was subjected to a Markov Cluster (MCL) algorithm analysis to determine orthologs groups using a default inflation value of 1.5 . A total of 2,873 and 2,874 coding genes were predicted by OrthoMCL in the chromosomic genomes of CFBP $8217^{\mathrm{T}}$ and LPPA $982^{\mathrm{T}}$, respectively. The number of core genes identified between the chromosomes of both strains was 2,857 while only one unique gene (FGI33_12335) encoding for a cysteine hydrolase was found in LPPA $982^{\mathrm{T}}$ (Figure 5A). In the orthologous analysis between the plasmids of both strains, 114 and 110 genes were found in LPPA $982^{\mathrm{T}}$ and CFBP $8217^{\mathrm{T}}$, respectively. The core gene sets contained between the plasmids were 107 whereas 3 CDS (FGI33_14985, FGI33_14995 and FGI33_15000 encoding for a helix-turn-helix transcriptional regulator, arsenate reductase ArsC and flavoprotein, respectively) were identified as unique genes in the plasmid of LPPA 
$982^{\mathrm{T}}$ (Figure 5A). The high number of orthologous genes observed between the genomes of strains CFBP $8217^{\mathrm{T}}$ and LPPA $982^{\mathrm{T}}$ corroborates not only that both strains belong to the same species but also that these two isolates seem to be clonal. All defined orthologous genes in the chromosome and plasmid of CFBP $8217^{\mathrm{T}}$ and LPPA982 ${ }^{\mathrm{T}}$ were illustrated in a Circos plot (Figure 5B) [43].

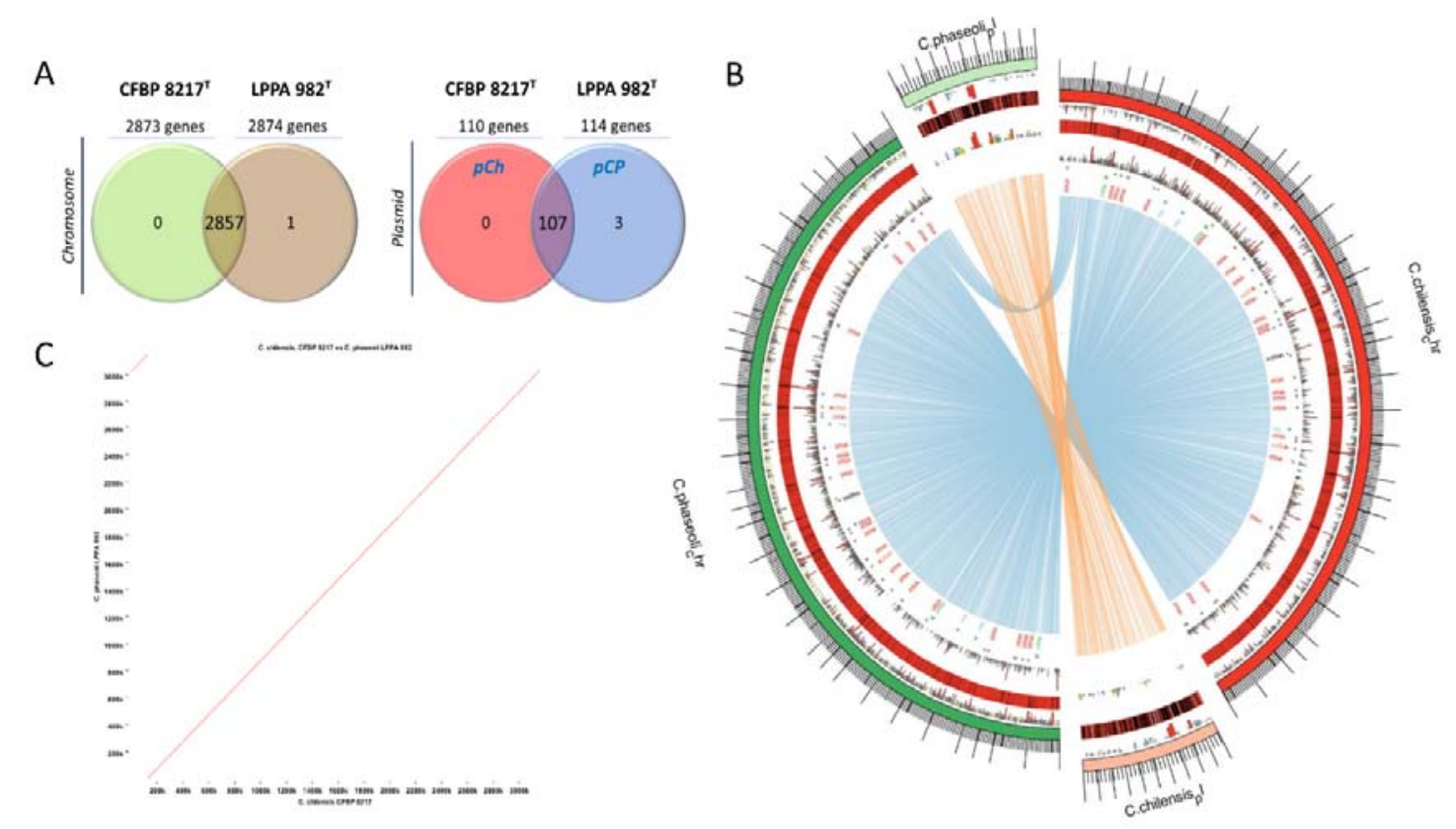

Figure 5. Orthologous analysis between C. michiganensis subsp. chilensis CFBP $8217^{\mathrm{T}}$ and C. michiganensis subsp. phaseoli LPPA $982^{\mathrm{T}}$. (A) Venn diagrams based on the core-genome generated from the OrthoMCL analysis between the chromosome and plasmid of CFBP $8217^{\mathrm{T}}$ and LPPA $982^{\mathrm{T}}$. The top panel over each circle indicates the total number of genes found in each strain at the chromosome and plasmid levels. The value located in the intersection of both circles shows the number of shared genes (core genes) between the chromosomes and plasmids of both strains. (B) Circos plot [43] illustrating the orthologous genes found between the chromosomes and plasmids of both strains. From the outermost to the innermost the layers portray: the genome coordinates (axis in $\mathrm{kb}$ ) of the chromosomes and plasmids; bar chart illustrating the sizes of those loci with a negative orientation; a red ring showing with black 
lines all coding sequences found in the chromosome and plasmid of each strain (the genes of the highest length can be visualized with a clear black line); bar chart illustrating the sizes of those loci with a positive orientation; text description pointing out the location of tRNA (red), rRNA (green), mRNA (black), ncRNA (orange) and regulatory genes (blue) in the chromosomes of both strains; and the inner layer depicting the connections among all homologous genes predicted between the chromosomes and plasmids of CFBP $8217^{\mathrm{T}}$ and LPPA $982^{\mathrm{T}}$. (C) in-silico syntenic dot plot showing a high similarity between the genomes of CFBP $8217^{\mathrm{T}}$ (x-axis) vs LPPA $982^{\mathrm{T}}$ (y-axis).

To further evaluate the genome similarities between the type strains of C. michiganensis subsp. chilensis and C. michiganensis subsp. phaseoli, an in-silico dot plot analysis was performed using the "Create Whole Genome Dot Plot" tool as implemented in the CLC Genomics Workbench v.21.0.5. The syntenic dot plot graphic (Figure 5C) displayed a clear collinear diagonal line between the sequences of strains CFBP $8217^{\mathrm{T}}$ and LPPA $982^{\mathrm{T}}$, indicating that the genomes of both strains are highly identical. This result emphasizes that strains CFBP $8217^{\mathrm{T}}$ and LPPA $982^{\mathrm{T}}$ must be considered as a single species.

\section{Conclusion}

To summarize, in the present study, we have sequenced the complete genomes of the type strains of the still-assigned subspecies C. michiganensis subsp. californiensis CFBP $8216^{\mathrm{T}}$, C. michiganensis subsp. chilensis CFBP $8217^{\mathrm{T}}$ and C. michiganensis subsp. phaseoli LPPA982 ${ }^{\mathrm{T}}$ along with C. tessellarius ATCC $33566^{\mathrm{T}}$ and C. nebraskensis A6096. This manuscript provides conclusive evidence to elevate the remaining three subspecies within the Clavibacter genus to the species level. By using different state-of-the-art in silico strategies such as MLSA, ANI, $\mathrm{dDDH}$, core-genome phylogenomics and whole-genome based ANI phylogeny, we have 
demonstrated that C. michiganensis subsp. californiensis must be raised to a species level for which we propose the name Clavibacter californiensis sp. nov. (type strain CFBP $8216^{\mathrm{T}}=\mathrm{ATCC}$ BAA-2691 $\left.{ }^{\mathrm{T}}=\mathrm{C} 55^{\mathrm{T}}\right) . \quad$ Likewise, different comparative genomic methods like orthologous and genome synteny analyses, as well as all above mentioned bioinformatic approaches, pointed out that type strains of C. michiganensis subsp. chilensis CFBP $8217^{\mathrm{T}}$ and C. michiganensis subsp. phaseoli LPPA982 ${ }^{\mathrm{T}}$ must be merged as a single organism and raised to a species level; consequently, we propose the name C. chilensis sp. nov. (type strain CFBP $8217^{\mathrm{T}}=$ ATCC BAA-2690 $\left.{ }^{\mathrm{T}}=\mathrm{ZUMM} 3936^{\mathrm{T}}\right)$. Additionally, we clarified the taxonomy description of a large panel composed of 80 Clavibacter strains. Our polyphasic analyses allowed us to determine that the ryegrass isolate AY1B2 is another strain of the proposed species C. californiensis sp. nov. while strains CFBP 7491 and VKM Ac-2886 isolated from tomato seeds and red elderberry, respectively, must be re-classified as C. chilensis sp. nov. Furthermore, we corroborated that the tomato endophyte strain CFBP 7576 constitutes another member of C. capsici. Interestingly, our high-throughput in-silico assessments revealed that the tulip strain CFBP 3399 belongs to the wheat pathogenic species, C. tessellarius. On the other hand, analysis indicated that strain DOAB 609 has been misnamed as C. tessellarius in the NCBI GenBank database, and should be described as a novel species of Clavibacter. In a similar way, the tomato endophytic strains CFBP 7494 and CFBP 8017 must be considered as a new species according to the calculated ANI and dDDH scores. Lastly, the TYGS allowed us to conclude that strains DOAB 609, CFBP 7494 and CFBP 8017 are certainly belong to new species. A further elaborated taxonomy study must be undertaken to provide the best fitting name for these potential novel species. 
Elevation in taxonomic rank from Clavibacter michiganensis subsp. californiensis (YasuharaBell and Alvarez, 2015) to Clavibacter californiensis (ca.li.for.ni.en'sis. N.L. masc. adj. californiensis pertaining to California, referring to the location of isolation of the type strain).

Basonym: Clavibacter michiganensis subsp. californiensis Yasuhara-Bell and Alvarez 2015.

The description of this taxon remains the same from its first description as Clavibacter michiganensis subsp. californiensis reported by Yasuhara-Bell and Alvarez, 2015.

The type strain is $\mathrm{C}^{\mathrm{T}} 5^{\mathrm{T}}\left(=\mathrm{CFBP} 8216^{\mathrm{T}}=\mathrm{ATCC}\right.$ BAA-2691 $)$ and it was isolated from seeds of Solanum lycopersycum in the state of California, USA. The DNA G+C content of the type strain was $72.78 \%$-while the DNA coding number of bases was $91.57 \%$.

\section{DESCRIPTION OF CLAVIBACTER CHILENSIS SP. NOV.}

Merging of Clavibacter michiganensis subsp. phaseoli (Gonzalez and Trapiello, 2014) and Clavibacter michiganensis subsp. chilensis (Yasuhara-Bell and Alvarez, 2015) as a single species and elevate to taxonomic level as Clavibacter chilensis (chil.en'sis. N.L. masc. adj. chilensis pertaining to Chile, referring to the location of isolation of the type strain).

Basonym: Clavibacter michiganensis subsp. chilensis Yasuhara-Bell and Alvarez 2015, Clavibacter michiganensis subsp. phaseoli Gonzalez and Trapiello 2014.

The description of this species is unchanged from its former information as Clavibacter michiganensis subsp. chilensis provided by Yasuhara-Bell and Alvarez, 2015.

The type strain is ZUM3936 ${ }^{\mathrm{T}}\left(=\mathrm{ATCC}\right.$ BAA-2690 $=$ CFBP $\left.8217^{\mathrm{T}}\right)$. The bacterium was isolated from seeds of Solanum lycopersycum from Chile. The DNA G+C content of the type strain is $73.43 \%$ whereas the DNA coding number of bases is $92.04 \%$. 


\section{AUTHOR STATEMENTS}

\section{Conflicts of interest}

The author(s) declare that there are no conflicts of interest

\section{Funding information}

This work was supported by the USDA National Institute of Food and Agriculture, Hatch project 9038H, managed by the College of Tropical Agriculture and Human Resources. Bioinformatic analysis tools were supported by NIGMS of the National Institutes of Health under award number P20GM125508. The strains were maintained by the grant from National Science Foundation (NSFCSBR Grant No. DBI-1561663).

\section{Ethical approval}

$N / A$

Consent for publication

$N / A$

\section{REFERENCES}

1. Davis MJ, Gillaspie AG, Vidaver AK, Harris RW. Clavibacter: a New Genus Containing Some Phytopathogenic Coryneform Bacteria, Including Clavibacter xyli subsp. xyli sp. nov., subsp. nov. and Clavibacter xyli subsp. cynodontis subsp. nov., Pathogens That Cause Ratoon Stunting Disease of Sugarcane and Bermudagrass Stunting Disease. International Journal of Systematic Bacteriology 1984;34:107-117.

2. Eichenlaub R, Gartemann K-H. The Clavibacter michiganensis subspecies: Molecular investigation of gram-positive bacterial plant pathogens. Annu Rev Phytopathol 2011;49:445-464.

3. Eichenlaub R, Gartemann K-H, Burger A. Clavibacter michiganensis, a group of grampositive phytopathogenic bacteria. In: Plant-Associated Bacteria. The Netherlands: Springer, Dordrecht; 2006. pp. 385-421. 
4. de León L, Siverio F, López MM, Rodríguez A. Clavibacter michiganesis subsp. michiganensis, a seedborne tomato pathogen: Healthy seeds are still the goal. Plant Disease 2011;95:1328-1338.

5. Lu Y, Ishimaru CA, Glazebrook J, Samac DA. Comparative Genomic Analyses of Clavibacter michiganensis subsp. insidiosus and Pathogenicity on Medicago truncatula. Phytopathology ${ }^{\circledast} 2018 ; 108: 172-185$.

6. Carlson RR, Vidaver AK. Taxonomy of Corynebacterium Plant Pathogens, Including a New Pathogen of Wheat, Based on Polyacrylamide Gel Electrophoresis of Cellular Proteins. International Journal of Systematic Bacteriology 1982;32:315-326.

7. Vidaver AK. Corynebacterium nebraskense, a New, Orange-Pigmented Phytopathogenic Species. International Journal of Systematic Bacteriology 1974;24:482-485.

8. Oh E-J, Bae C, Lee H-B, Hwang IS, Lee H-I, et al. Clavibacter michiganensis subsp. capsici subsp. nov., causing bacterial canker disease in pepper. International Journal of Systematic and Evolutionary Microbiology 2016;66:4065-4070.

9. González AJ, Trapiello E. Clavibacter michiganensis subsp. phaseoli subsp. nov., pathogenic in bean. International Journal of Systematic and Evolutionary Microbiology 2014;64:1752-1755.

10. European and Mediterranean Plant Protection Organization (EPPO). PM 1/2 (30) EPPO A1 and A2 Lists of pests recommended for regulation as quarantine pests. 21 Boulevard Richard Lenoir, 75011 Paris, France. https://gd.eppo.int/taxon/CORBIN/documents (September 2021). 
11. Yasuhara-Bell J, Alvarez AM. Seed-associated subspecies of the genus Clavibacter are clearly distinguishable from Clavibacter michiganensis subsp. michiganensis. International Journal of Systematic and Evolutionary Microbiology 2015;65:811-826.

12. Li X, Tambong J, Yuan K (Xiaoli), Chen W, Xu H, et al. Re-classification of Clavibacter michiganensis subspecies on the basis of whole-genome and multi-locus sequence analyses. International Journal of Systematic and Evolutionary Microbiology $2018 ; 68: 234-240$.

13. Nouioui I, Carro L, García-López M, Meier-Kolthoff JP, Woyke T, et al. Genome-Based Taxonomic Classification of the Phylum Actinobacteria. Front Microbiol 2018;9:2007.

14. Tian Q, Chuan J, Sun X, Zhou A, Wang L, et al. Description of Clavibacter zhangzhiyongii sp. nov., a phytopathogenic actinobacterium isolated from barley seeds, causing leaf brown spot and decline. International Journal of Systematic and Evolutionary Microbiology;71. Epub ahead of print 13 May 2021. DOI: 10.1099/ijsem.0.004786.

15. Hugenholtz P, Chuvochina M, Oren A, Parks DH, Soo RM. Prokaryotic taxonomy and nomenclature in the age of big sequence data. ISME J 2021;15:1879-1892.

16. Kämpfer P, Glaeser SP. Prokaryotic taxonomy in the sequencing era - the polyphasic approach revisited: Prokaryotic taxonomy in the sequencing era. Environmental Microbiology 2012;14:291-317.

17. Chung M, Munro JB, Tettelin H, Hotopp JCD. Using Core Genome Alignments To Assign Bacterial Species. 2018;3:21. 
18. Ciufo S, Kannan S, Sharma S, Badretdin A, Clark K, et al. Using average nucleotide identity to improve taxonomic assignments in prokaryotic genomes at the NCBI. International Journal of Systematic and Evolutionary Microbiology 2018;68:2386-2392.

19. Richter M, Rosselló-Móra R. Shifting the genomic gold standard for the prokaryotic species definition. PNAS 2009;106:19126-19131.

20. Goris J, Konstantinidis KT, Klappenbach JA, Coenye T, Vandamme P, et al. DNA-DNA hybridization values and their relationship to whole-genome sequence similarities. International Journal of Systematic and Evolutionary Microbiology 2007;57:81-91.

21. Auch AF, von Jan M, Klenk H-P, Göker M. Digital DNA-DNA hybridization for microbial species delineation by means of genome-to-genome sequence comparison. Standards in Genomic Sciences 2010;2:117-134.

22. Osdaghi E, Portier P, Briand M, Taghouti G, Jacques M-A. Draft Genome Sequences of the Type Strains of Three Clavibacter Subspecies and Atypical Peach-Colored Strains Isolated from Tomato. Microbiol Resour Announc;7. Epub ahead of print 8 November 2018. DOI: 10.1128/MRA.01357-18.

23. Osdaghi E, Rahimi T, Taghavi SM, Ansari M, Zarei S, et al. Comparative Genomics and Phylogenetic Analyses Suggest Several Novel Species within the Genus Clavibacter, Including Nonpathogenic Tomato-Associated Strains. Appl Environ Microbiol;86. Epub ahead of print 2 March 2020. DOI: 10.1128/AEM.02873-19.

24. Méndez V, Valenzuela M, Salvà-Serra F, Jaén-Luchoro D, Besoain X, et al. Comparative Genomics of Pathogenic Clavibacter michiganensis subsp. michiganensis Strains from 
Chile Reveals Potential Virulence Features for Tomato Plants. Microorganisms 2020;8:1679.

25. Thapa SP, Pattathil S, Hahn MG, Jacques M-A, Gilbertson RL, et al. Genomic Analysis of Clavibacter michiganensis Reveals Insight Into Virulence Strategies and Genetic Diversity of a Gram-Positive Bacterial Pathogen. MPMI 2017;30:786-802.

26. Tatusova T, DiCuccio M, Badretdin A, Chetvernin V, Nawrocki EP, et al. NCBI prokaryotic genome annotation pipeline. Nucleic Acids Res 2016;44:6614-6624.

27. Huntemann M, Ivanova NN, Mavromatis K, Tripp HJ, Paez-Espino D, et al. The standard operating procedure of the DOE-JGI Microbial Genome Annotation Pipeline (MGAP v.4). Stand in Genomic Sci 2015;10:86.

28. Chen I-MA, Chu K, Palaniappan K, Pillay M, Ratner A, et al. IMG/M v.5.0: an integrated data management and comparative analysis system for microbial genomes and microbiomes. Nucleic Acids Research 2019;47:D666-D677.

29. Brettin T, Davis JJ, Disz T, Edwards RA, Gerdes S, et al. RASTtk: A modular and extensible implementation of the RAST algorithm for building custom annotation pipelines and annotating batches of genomes. Sci Rep 2015;5:8365.

30. Konstantinidis KT, Tiedje JM. Genomic insights that advance the species definition for prokaryotes. Proceedings of the National Academy of Sciences 2005;102:2567-2572.

31. Meier-Kolthoff JP, Auch AF, Klenk H-P, Göker M. Genome sequence-based species delimitation with confidence intervals and improved distance functions. $B M C$ Bioinformatics 2013;14:60. 
32. Meier-Kolthoff JP, Carbasse JS, Peinado-Olarte RL, Göker M. TYGS and LPSN: a database tandem for fast and reliable genome-based classification and nomenclature of prokaryotes. Nucleic Acids Research 2021;gkab902.

33. Meier-Kolthoff JP, Auch AF, Klenk H-P, Göker M. Genome sequence-based species delimitation with confidence intervals and improved distance functions. BMC Bioinformatics 2013;14:60.

34. Załuga J, Stragier P, Baeyen S, Haegeman A, Van Vaerenbergh J, et al. Comparative genome analysis of pathogenic and non-pathogenic Clavibacter strains reveals adaptations to their lifestyle. BMC Genomics 2014;15:392.

35. Zaluga J, Van Vaerenbergh J, Stragier P, Maes M, De Vos P. Genetic diversity of nonpathogenic Clavibacter strains isolated from tomato seeds. Systematic and Applied Microbiology 2013;36:426-435.

36. Jacques M-A, Durand K, Orgeur G, Balidas S, Fricot C, et al. Phylogenetic Analysis and Polyphasic Characterization of Clavibacter michiganensis Strains Isolated from Tomato Seeds Reveal that Nonpathogenic Strains Are Distinct from C. michiganensis subsp. michiganensis. Appl Environ Microbiol 2012;78:8388-8402.

37. Seemann T. Prokka: rapid prokaryotic genome annotation. Bioinformatics 2014;30:20682069.

38. Page AJ, Cummins CA, Hunt M, Wong VK, Reuter S, et al. Roary: rapid large-scale prokaryote pan genome analysis. Bioinformatics 2015;31:3691-3693. 
39. Löytynoja A. Phylogeny-aware alignment with PRANK. In: Russell DJ (editor). Multiple Sequence Alignment Methods. Totowa, NJ: Humana Press. pp. 155-170.

40. Kozlov AM, Darriba D, Flouri T, Morel B, Stamatakis A. RAxML-NG: a fast, scalable and user-friendly tool for maximum likelihood phylogenetic inference. Bioinformatics 2019;35:4453-4455.

41. Meier-Kolthoff JP, Göker M. TYGS is an automated high-throughput platform for stateof-the-art genome-based taxonomy. Nat Commun 2019;10:2182.

42. Li L, Stoeckert CJ, Roos DS. OrthoMCL: Identification of Ortholog Groups for Eukaryotic Genomes. Genome Res 2003;13:2178-2189.

43. Krzywinski M, Schein J, Birol i, Connors J, Gascoyne R, et al. Circos: An information aesthetic for comparative genomics. Genome Res 2009;19:1639-1645.

\section{FIGURES AND TABLES}

Figure 1. Combined average nucleotide identity (ANI, upper diagonal) and digital DNA-DNA hybridization (dDDH, lower diagonal) heatmap among all Clavibacter species. Cut-off values for species delineation are $96 \%(\mathrm{ANI})$ and $70 \%(\mathrm{dDDH})$.

Figure 2. Multi-Locus Sequence Analysis (MLSA) based on the concatenated alignment of seven housekeeping genes, acnA, atpD, bipA, icdA, mtlD, recA, and rpoB. The maximumlikelihood (ML) phylogenetic tree was created using 80 Clavibacter strains with a bootstrap test of 1,000 replicates. Bootstrap values are indicated at branch points. Evolutionary distances were estimated by applying the Maximum Composite Likelihood (MCL) approach. There was 
a total of 13,154 positions (alignment length) in the final dataset. The tree was drawn to scale, with branch lengths measured in the number of substitutions per site. The branches were colorcoded to emphasize the clusters of each species. The genomes of the five Clavibacter strains sequenced in this study are pointed out with a teal diamond. Rathayibacter iranicus NCPPB 2253 and $R$. toxicus WAC3373 served as outgroups to root the tree. The evolutionary analysis was conducted in MEGA 11.

Figure 3. Maximum-likelihood (ML) phylogeny generated from the core-genome of 78 Clavibacter strains. (A) Pie chart depicting the proportion of core and accessory genes among the 78 Clavibacter genomes as predicted by the ROARY pipeline. (B) ML phylogentic tree reconstructed from the concatenated alignment of 1,028 core genes. A bootstrap test of 1,000 replicates was used to assess the statistical support of each node. The ML analysis was performed using the RAxML-NG tool with a General Time-Reversible GAMMA model. The phylogenetic tree was mid-point rooted and illustrated based on the increasing order of nodes. All strains and their respective branches of each Clavibacter species are highlighted with specific colors. The proposed species are pinpointed in a black rectangular frame. FigTree v1.4.4 was used to visualize and color-coded the ML tree.

Figure 4. Whole-genome phylogeny based on the computed average nucleotide identity (ANI) of 79 Clavibacter strains. The neighbor joining tree was created using the CLC Genomics Workbench v.21.0.5 with a bootstrap test of 100 replicates. Rathayibacter toxicus WAC3373 was used as an outgroup to root the tree. The legends of layers corresponding to the organism and host, along with the node shape, are indicated on the left side of the graphic. 
Figure 5. Orthologous analysis between C. michiganensis subsp. chilensis CFBP $8217^{\mathrm{T}}$ and C. michiganensis subsp. phaseoli LPPA $982^{\mathrm{T}}$. (A) Venn diagrams based on the core-genome generated from the OrthoMCL analysis between the chromosome and plasmid of CFBP $8217^{\mathrm{T}}$ and LPPA $982^{\mathrm{T}}$. The top panel over each circle indicates the total number of genes found in each strain at the chromosome and plasmid levels. The value located in the intersection of both circles shows the number of shared genes (core genes) between the chromosomes and plasmids of both strains. (B) Circos plot [43] illustrating the orthologous genes found between the chromosomes and plasmids of both strains. From the outermost to the innermost the layers portray: the genome coordinates (axis in $\mathrm{kb}$ ) of the chromosomes and plasmids; bar chart illustrating the sizes of those loci with a negative orientation; a red ring showing with black lines all coding sequences found in the chromosome and plasmid of each strain (the genes of the highest length can be visualized with a clear black line); bar chart illustrating the sizes of those loci with a positive orientation; text description pointing out the location of tRNA (red), rRNA (green), mRNA (black), ncRNA (orange) and regulatory genes (blue) in the chromosomes of both strains; and the inner layer depicting the connections among all homologous genes predicted between the chromosomes and plasmids of CFBP $8217^{\mathrm{T}}$ and LPPA $982^{\mathrm{T}}$. (C) in-silico syntenic dot plot showing a high similarity between the genomes of CFBP $8217^{\mathrm{T}}$ (x-axis) vs LPPA $982^{\mathrm{T}}$ (y-axis). 
Table 1. Genome features and assembly statistics for the five Clavibacter strains sequenced in this study.

\begin{tabular}{|c|c|c|c|c|c|}
\hline Genomic Features & $\begin{array}{l}\text { C. michiganensis subsp. } \\
\text { californiensis CFBP } 8216^{\top}\end{array}$ & $\begin{array}{c}\text { C. michiganensis } \\
\text { subsp. chilensis CFBP } \\
8217^{\top}\end{array}$ & $\begin{array}{c}\text { C. michiganensis } \\
\text { subsp. phaseoli LPPA } \\
9^{982^{\top}}\end{array}$ & $\begin{array}{c}\text { C. } \\
\text { tessellarius } \\
\text { ATCC } 33566^{\top}\end{array}$ & $\begin{array}{c}\text { C. nebraskensis } \\
\text { A6096 }\end{array}$ \\
\hline GenBank accession no. & СР040792-СР040794 & СР040795-СР040796 & СР040786-СР040787 & $\begin{array}{l}\text { CP040788- } \\
\text { СР040791 }\end{array}$ & СР040797 \\
\hline GOLD project ID in IMG database & Gp0434066 & Gp0434065 & Gp0432876 & Gp0432876 & Gp0434073 \\
\hline Assembly level & Complete & Complete & Complete & Complete & Complete \\
\hline Genome coverage $(x)$ & $712.0 x$ & $373.0 x$ & $468.0 x$ & $574.0 x$ & $289.0 x$ \\
\hline Genome size (bp) & 3243492 & 3221227 & 3226754 & 3329338 & 3069018 \\
\hline $\mathrm{G}+\mathrm{C}$ content $(\mathrm{mol} \%)^{\varphi}$ & $72.78 \%$ & $73.43 \%$ & $73.42 \%$ & $73.63 \%$ & $72.97 \%$ \\
\hline Chromosome length (bp) & 3165486 & 3103717 & 3103693 & 3254008 & 3069018 \\
\hline Plasmid length (bp)* & 78006 (pCCa) & 117510 (pCh) & 123061 (pCP) & $\begin{array}{l}29539 \text { (рСТ1) } \\
45791 \text { (рСТ2) }\end{array}$ & - \\
\hline Genes (total) & 3086 & 3070 & 3075 & 3199 & 2938 \\
\hline Coding sequences (CDS) & 3032 & 3016 & 3021 & 3145 & 2884 \\
\hline $\begin{array}{l}\text { Protein coding genes with function } \\
\text { prediction } \varphi\end{array}$ & 2337 & 2397 & 2402 & 2375 & 2229 \\
\hline Protein coding genes with enzymes ${ }^{\varphi}$ & 871 & 870 & 874 & 883 & 858 \\
\hline $\begin{array}{l}\text { Protein coding genes connected to } \\
\text { KEGG pathways }{ }^{\varphi}\end{array}$ & 935 & 930 & 932 & 937 & 901 \\
\hline Protein coding genes with COGs ${ }^{\varphi}$ & 2352 & 2420 & 2425 & 2401 & 2256 \\
\hline rRNAs $(5 S, 16 S, 23 S)$ & $2,2,2$ & $2,2,2$ & $2,2,2$ & $2,2,2$ & $2,2,2$ \\
\hline tRNAs & 45 & 45 & 45 & 45 & 45 \\
\hline ncRNAs & 3 & 3 & 3 & 3 & 3 \\
\hline Pseudogenes & 67 & 32 & 29 & 100 & 83 \\
\hline
\end{tabular}

${ }^{\varphi}$ Genomic features as annotated using the Integrated Microbial Genomes annotation pipeline v.5.0.3 from the Joint Genome Institute (IMG-JGI).

*Assigned name for the plasmids is indicated between parentheses.

GOLD: Genomes OnLine Database; KEGG: Kyoto Encyclopedia of Genes and Genomes; COG: Cluster of Orthologous Groups of proteins; rRNA: ribosomal RNA; tRNA: transfer RNA; ncRNA: non-coding RNA. 


\section{SUPPLEMENTARY DATA}

Table S1. Detailed list of genomic information of the 76 Clavibacter strains retrieved from the NCBI GenBank database and the five Clavibacter genomes sequenced in this study (highlighted in light blue color). The strains presented in this table were used for different in silico analyses such as MLSA, ANI and $d D D H$ computation, reconstructed ML phylogenomic tree from core-genome alignment, ANI-based phylogeny, and taxonomy assessment of undefined species in the TYGS platform.

Table S2. Pairwise heatmap comparison based on the ANI computation values among the 79 Clavibacter strains, including the five strains sequenced in this study. The ANI scores were determined in the CLC Genomics Workbench 21.0.5. Strain CFBP $2404^{\mathrm{T}}$ was not included in the analysis since this isolate is the same type strain LMG $3663^{\mathrm{T}}$

Table S3. Pairwise heatmap comparison based on the $\mathrm{dDDH}$ computation values among the 79 Clavibacter strains, including the five strains sequenced in this study. The dDDH scores were calculated in the web server Genome-to-Genome Distance Calculator (GGDC) version 3.0 (http://ggdc.dsmz.de/ggdc.php\#). Strain CFBP $2404^{\mathrm{T}}$ was not included in the analysis since this isolate is the same type strain LMG $3663^{\mathrm{T}}$ 